\title{
Mathematical Anti-Realism and Explanatory Structure
}

\author{
Bruno Whittle
}

January 27,2021

When our metaphysical tools are defined in modal terms, there is a limit to how much we can offer to the study of mathematics. For example, since any (pure) mathematical facts strictly imply (i.e. entail) any other, it seems that such a notion will not get us very far in this area. If, however, we can lay on our hands on some more discerning tools, which can prize apart facts with a common modal profile, then there is hope that we can say something more illuminating. This paper is an attempt to take an initial step in the direction of realizing this hope. ${ }^{1}$ In particular, I focus on a plausible, and popular, general approach to mathematics, which I term 'paraphrase anti-realism'. And I examine this with the aid of the notion of grounding; the notion, that is, of metaphysical explanation. The results of this examination are surprising and, I believe, shed light on the approach.

The position of paraphrase anti-realism, then, can be introduced as follows. It is plausible that standard mathematical claims, such as 'there are infinitely many primes' or 'every consistent set of sentences of has a model', are true; but that, nevertheless, if one rifles through the fundamental furniture of the world, one will not find amongst this mathematical objects, such as numbers, sets or models. The natural way of making sense of this would seem to be with the thought: this is possible, because such standard claims have paraphrases that make clear how their truth does not require the fundamental existence of mathematical objects. The aim of this paper is to tease out some consequences of this approach for explanatory structure, or, more precisely, for the relation between explanatory structure and logical structure.

It is tempting to think that this relation is straightforward. Thus, conjunctions and disjunctions would seem to be explained, or grounded, by their (true) immedi-

\footnotetext{
${ }^{1}$ I do not however claim that it is the first such step: see, e.g., Rosen [2011].
} 
ate components, while generalizations are grounded by their (true) instances. For example, snow is black or white because it is black, and something is white for the same reason. Indeed, such examples of logically complex claims being grounded by logically simpler ones are typically regarded as paradigms of the notion of metaphysical explanation, used to introduce it in the first place. And, of course, this simple story relating logical and explanatory structure seems as obvious in the case of mathematics as elsewhere. Thus, it can seem undeniable that 17 or 18 is odd because 17 is, or that every natural number greater than 1 has a unique prime decomposition in part because 42 does, and so on. I argue, however, that paraphrase anti-realism fundamentally rewrites this story. Not only do these basic claims relating logical and explanatory structure fail on this approach; there are even cases where they are reversed. For example, there are cases where generalizations explain their instances, or disjunctions their disjuncts.

The primary conclusion of the paper is simply that paraphrase anti-realism has this apparently striking consequence for explanatory structure. But one reason this seems significant-and why it might seem to constitute an objection to this approach-is as follows. In general, anti-realist takes on mathematics are of course intended to challenge our naive conception of this. But those that are our focus here, which take as their starting point the idea that standard mathematical claims are true, seem to want to challenge this only insofar as it concerns the relation of mathematics to fundamental reality. The idea is thus that while certain 'external' claims about mathematics — claims about how mathematics relates to fundamental reality-must be given up, 'internal' claims_claims about mathematical reality in and of itself-are to be left untouched. Claims about which mathematical facts hold in virtue of which others, however, seem very clearly to be on the 'internal' side of this division. They are a basic part of our understanding of mathematical reality, in and of itself, it seems. And so one might hope, or even expect, that paraphrase anti-realism would preserve such explanatory claims.

I argue then that there are, for example, general facts, along the lines of every number has a successor that, under paraphrase anti-realism, are not grounded by their instances; indeed, quite the reverse. The argument proceeds by unpacking such generalizations into their paraphrases, and using these to locate them in the explanatory structure. I should acknowledge at the outset, however, that there is a sense in which (from the perspective of paraphrase anti-realism) claims such as 'every number has a successor' are not 'ultimately' generalizations: from the internal mathematical perspective they are generalizations, but the underlying paraphrases have a different logical form. There is thus a sense in which, for all that I will argue, the anti-realist approaches in question are compatible with the standard principles 
relating logical and explanatory structure: i.e. with claims whose underlying form is that of a generalization being grounded by their instances, and so on.

Some readers might worry that this undercuts the significance of the paper's conclusion, or, at least, blunts its force as an objection. ${ }^{2}$ For (the line of thought would run) the only reason to think that every number's having a successor is grounded by o having a successor, 1 having a successor, etc. is that we take the former claim to be a universal generalization. But if our position is that this claim is not ultimately (i.e. when unpacked into its paraphrase) universal, then we should be quite happy to give up on its being grounded by its (apparent) instances.

I think this worry is misplaced, however. The idea behind paraphrase antirealism, as I have said, seems to be to preserve our internal judgements about mathematics, while revising our external ones (concerning the relation of mathematics to fundamental reality), and judgements about which mathematical facts explain which others seem firmly to be on the internal side of this divide. An analogy might help to drive this point home. From the perspective of paraphrase anti-realism, there is a sense in which, for example, Goldbach's conjecture (i.e. every even number greater than two is the sum of two primes) is not ultimately universal. Thus, one could in principle say that the conjecture is false, despite the fact that 4 is the sum of two primes, 6 is the sum of two primes etc. - while at the same time maintaining (in the manner envisaged) that every universal is true iff its instances are. Nevertheless, it would surely be unattractive to say that Goldbach's conjecture is false, while these latter claims are true, precisely because the idea that such (apparent) universals are true iff their instances are would seem to be a basic aspect of our understanding of mathematics. And the view that Goldbach's conjecture is true, without being grounded by 4 being the sum of two primes, by 6 being etc., would seem to be similarly unattractive, even if this is compatible with (ultimate) universals always being grounded by their instances; because, in this case too, the relationship between these claims would seem to be a basic part of our understanding of mathematical reality.

The structure of the paper is as follows. In $\$ 1$ I sketch a number of strategies for providing paraphrases of mathematical claims. The aim is to show how a whole family of approaches give rise to reversals of the commonly assumed logicoexplanatory claims. So I do not discuss any particular extant approach in detail. I do however indicate where proposals along the lines of those I consider have been put forward: and it would be straightforward to apply the arguments of the paper to these extant proposals. In $\$ 2$ I then draw out the consequences, for the relationship

\footnotetext{
${ }^{2}$ I am grateful to a referee for raising this.
} 
between logical and explanatory structure, of the strategies that I have presented. Finally, in $\$_{3} I$ discuss a number of issues arising from the arguments of the paper.

\section{Paraphrase Strategies}

How might one supply mathematical claims with paraphrases? Here is a natural idea. 'The number of planets is even', for example, is true, despite the failure of numbers to fundamentally exist, because all that one is really saying is: given the assumption that there are numbers, the one of these that corresponds to the planets is even. Or to put it even more simply: if there are numbers, then that which corresponds to the planets is even.

In this section I give a number of strategies for making this basic idea more precise. For the purposes of illustration, and for much of the paper, I focus on claims that are about (natural) numbers together with non-mathematical objects, but that are not about any other mathematical objects. However, one will presumably want to give a uniform account of mathematical paraphrases, and so if one pursues one of the strategies below for claims about numbers, then one will also use that strategy for claims about other mathematical objects, such as real numbers or sets. And, indeed, everything that I say about numerical claims can be straightforwardly adapted to apply to claims about other sorts of mathematical objects.

The basic idea that we want to make precise, then, is that the paraphrase of 'the number of planets is even' is along the lines: if there are numbers, then that which corresponds to the planets is even. In all of the strategies I consider for how to do this, the bare existential 'there are numbers' is replaced with something more informative, namely, a standard set of axioms about numbers. For this purpose, I use second-order Peano arithmetic (PA2). ${ }^{3}$ Such a replacement would seem essential. For in talking about numbers we of course rely not merely on the assumption that they exist, but on all sorts of further assumptions about them as well. But then these further assumptions should also be built into our paraphrases. And the natural way of doing that would seem to be by using a set of axioms from which these assumptions can be derived. Further, there are a range of reasons for using PA2 in particular. First, it seems to be the theory that best captures our 'folk theory' of number. ${ }^{4}$ Second, it is complete. ${ }^{5}$ If we instead used an incomplete theory, such as

\footnotetext{
${ }^{3}$ The axioms of $\mathrm{PA} 2$ are listed in the appendix.

${ }^{4}$ See, e.g., Dedekind [1888].

${ }^{5}$ That is, for any sentence $\alpha$ of the language of PA2, either $\alpha$ or $\neg \alpha$ is a logical consequence of PA2.
} 
first-order Peano arithmetic (PA1), then true but unprovable sentences, such as the Gödel sentence of PA1, would lead to counterexamples to at least some of the proposals below. Third, PA2 is finite, which makes our life easier since it means that we do not need a 'trick' to deal with infinitely many sentences, such as a truth predicate, infinitary conjunction or quantification into sentence position. Having said all of that, however, if for some reason one preferred to do things in terms of some alternative set of axioms, then the arguments of the paper could straightforwardly enough be adapted to that version of the strategies below.

Here then are the strategies. In giving these, I let $\alpha$ be some arbitrary claim about numbers and non-mathematical objects: e.g. 'the number of planets is even' or 'there are infinitely many primes'.

\subsection{Consequence Strategy}

According to this, what we are really saying when we assert $\alpha$ is: if our basic axioms about numbers hold, together with 'the facts', then it follows logically that $\alpha$. Here 'the facts' means the facts about non-mathematical objects.

To state this more precisely we need two things: a way of talking about logical consequence, and a way of talking about the facts. To this end, I use $\Rightarrow$ as a logical consequence connective. So $\beta \Rightarrow \gamma$ means: if $\beta$, then it follows logically that $\gamma$. Thus $\beta \Rightarrow \gamma$ is equivalent to: $\{\beta\} \vDash \gamma$. But using a connective seems preferable, since it yields a paraphrase that uses rather than mentions the relevant sentences. Surely, when we make claims about numbers, we are not in fact making claims about sentences about numbers. Further, I take $\Rightarrow$ to be multigrade in its first argument, i.e. for any $n \geq 1$ and $\beta_{1}, \ldots, \beta_{n},\left(\beta_{1}, \ldots, \beta_{n}\right) \Rightarrow \gamma$ is well formed.

I assume that there is some finite set of sentences $\mathcal{F}$ whose members express precisely the facts. One could get by without the assumption that $\mathcal{F}$ is finite (just as one could use an infinite set of axioms instead of PA2), but I make this because it simplifies things. Now let $C$ be a new unary predicate symbol (i.e. not occurring in PA2 or $\mathcal{F}$ ), intended to mean: is concrete. For a formula $\varphi, \varphi_{C}$ is the result of restricting the quantifiers in $\varphi$ by $C{ }^{6}$ And $\mathcal{F}_{C}$ is $\left\{\beta_{C}: \beta \in \mathcal{F}\right\}$. We give the paraphrase in terms of $\mathcal{F}_{C}$ rather than $\mathcal{F}$ since we want to consider the result of combining the facts with our mathematical claims (i.e. PA2). But $\mathcal{F}$ might be inconsistent with PA2: for example, if $\mathcal{F}$ contains a sentence only true in finite models, such as $\forall x\left(x=b_{1} \vee \cdots \vee x=b_{n}\right)$. Such problems are avoided by moving to $\mathcal{F}_{C}$, since if

\footnotetext{
${ }^{6}$ More explicitly, if $\varphi$ is atomic, then $\varphi_{C}$ is simply $\varphi$; if $\varphi$ is $\neg \psi$, then $\varphi_{C}$ is $\neg \psi_{C}$; if $\varphi$ is $\psi \rightarrow \chi$, then $\varphi_{C}$ is $\psi_{C} \rightarrow \chi_{C}$; while if $\varphi$ is $\forall x \psi$, then $\varphi_{C}$ is $\forall x\left(C x \rightarrow \psi_{C}\right)$.
} 
$\beta \in \mathcal{F}$ is the sentence just mentioned, then $\mathcal{F}_{C}$ will only contain $\beta_{C}$, i.e.

$$
\forall x\left(C x \rightarrow x=b_{1} \vee \cdots \vee x=b_{n}\right),
$$

which is perfectly consistent with PA2. Finally, if $\beta_{1}, \ldots, \beta_{n}$ are the members of $\mathcal{F}_{C} \cup \mathrm{PA} 2$, then I use $\mathcal{M}$ as shorthand for this list. The paraphrase of $\alpha, \alpha_{P}$, that this strategy proposes is then essentially: $\mathcal{M} \Rightarrow \alpha$.

There is however one further wrinkle. The strategies of this section rely on claims about numbers leading a kind of 'double life'. On the one hand, they have a 'superficial', or 'everyday', use, on which they do not-the idea is-require the fundamental existence of numbers. It is this use that the strategies are giving an account of. On the other hand, however, these strategies rely on claims about numbers also having a fundamental use, on which 'there are infinitely many primes', for example, does require the fundamental existence of numbers. This is how we are using claims about numbers when they occur within our paraphrases. For it would seem circular to rely on the superficial use of $\alpha$ in giving an account of what that very use of $\alpha$ amounts to. ${ }^{7}$

Indeed, quite independently of the specific strategies of this section, it is plausible that on the general anti-realist approach to mathematics under consideration, numerical claims will have these two distinct uses. For while we want to say that 'there are infinitely many primes' is true in everyday or mathematical contexts, nevertheless (the approach maintains), numbers do not fundamentally exist. But then, when our aim is to describe how things are fundamentally, it seems rather to be correct to say: there are no numbers (and a fortiori no primes).

For clarity, I use bold symbols for the fundamental use, and regular ones for the superficial use. ${ }^{8}$ More precisely, then, the paraphrase proposed by this strategy, $\alpha_{P}$, is

$$
\mathcal{M} \Rightarrow \boldsymbol{\alpha} .
$$

An alternative version of this idea would replace the notion of logical consequence with that of strict implication, i.e. $\Rightarrow$ would be replaced by $\square\left({ }_{-} \rightarrow_{-}\right) .9$

\footnotetext{
${ }^{7}$ In fact, this circularity worry is more pressing in the case of the subsequent strategies of this section: since it is at least arguable that one can know about the merely logical behaviour of numerical claims (under the superficial use) without knowing what this use amounts to (i.e. which paraphrases capture it). However, for the sake uniformity, I give all of the strategies in terms of the fundamental use.

${ }^{8}$ Thus, officially, our language contains two distinct sentences, $\boldsymbol{\alpha}$ and $\alpha$, where the former corresponds to the fundamental use and the latter to the superficial one.

${ }^{9}$ For attempts to use paraphrases along these lines to elucidate standard mathematical claims, see Putnam [1967] and Hellman [1989, 1996].
} 
However, since it would seem that if a numerical claim $\beta$ is false, then it is necessarily so, this version of the strategy requires PA2 and $\boldsymbol{\alpha}$ to be generalized, i.e. non-logical symbols would be replaced by variables, and the whole conditional would be universally quantified. Thus, for simplicity I focus on the version of the strategy in terms of logical consequence. But similar points could be made about this alternative.

\subsection{Counterfactual Strategy}

According to this, when we say that there are infinitely many primes, what we are really saying is that if there were numbers, then there would be so many primes. I use $\square \rightarrow$ for the counterfactual conditional, and, as with $\Rightarrow$, I take this to be multigrade in its first argument. Thus ${ }^{10}$

$$
\alpha_{P}: \mathbf{P A 2} \square \rightarrow \boldsymbol{\alpha} .
$$

We do not need $\mathcal{F}_{C}$ in this case. For what $\beta \square \leftrightarrow \gamma$ means is essentially: in the situation in which $\beta$ is true, but that is in other respects as close as possible to the actual situation, $\gamma$ is also true. But the situation in which PA2 is true, yet which is in other respects as close as possible to actuality, will be one in which the facts about non-mathematical objects, i.e. $\mathcal{F}_{C}$, also obtain.

Obviously, this strategy requires that we can make sense of counterpossibles ${ }^{11}$. That is, it requires that we do not classify all of these as vacuously true. (If all such counterfactuals are true, then on this proposal so is 'there are not infinitely many primes', for example.) Fortunately, it seems clear that in this sort of case in particular we can indeed make sense of such counterfactuals. For example, it seems clear that while (1) and (3) are true, (2) and (4) are not. Thus, given the plausible assumption that the antecedents are all either necessary or impossible, at least one of (2) and (4) is a false counterpossible.

(1) If PA2 were the case (i.e. if there were numbers and they satisfied the standard axioms), then there would infinitely many primes.

(2) If PA2 were the case, then there would be no primes between 20 and 30.

(3) If there were no numbers, then there would be no primes.

(4) If there were no numbers, then there would still be a prime between 20 and 30.

\footnotetext{
${ }^{10}$ For this proposal, see Dorr [2008].

${ }^{11}$ I.e. counterfactuals with metaphysically impossible antecedents.
} 


\subsection{Fiction Strategy}

The idea behind the final strategy is that when we make a numerical claim what we are really saying is that this holds in the number fiction. The natural way to make this precise is to think of fictions as lists of sentences, and to use an operator $\square_{G}$ such that $\square_{G} \beta$ means: according to the fiction that $G, \beta$. The proposed paraphrase is then ${ }^{12}$

$$
\alpha_{P}: \square_{\mathcal{M}} \boldsymbol{\alpha}
$$

\section{Logical vs Explanatory Structure}

Suppose that one of these strategies for giving paraphrases of mathematical claims is correct. What are the consequences for the relationship between logical and explanatory structure in this realm? To discuss explanation, I use the notion of partial ground, and I write $\beta<\gamma$ to mean that $\beta$ is a partial ground of $\gamma \cdot{ }^{13}$ It is tempting to think that the following 'logico-explanatory' (LE) principles hold. Here $\beta$ and $\gamma$ are sentences, $\varphi$ is a formula with at most $x$ free, and $t$ is a closed term. ${ }^{14}$

$(\wedge \mathrm{L})$ If $\beta \wedge \gamma$, then $\beta<\beta \wedge \gamma$.

$(\wedge \mathrm{R})$ If $\beta \wedge \gamma$, then $\gamma<\beta \wedge \gamma$.

( $\vee L)$ If $\beta$, then $\beta<\beta \vee \gamma$.

\footnotetext{
${ }^{12}$ For a proposal along these lines, see Chihara [1973]. (Rosen [1990] gives a similar treatment of claims about possible worlds.) Alternative fictionalist approaches to mathematics are given by Field [1989: 1-52] and Yablo [2001]. The latter are not versions of paraphrase anti-realism in the sense of this paper: for one thing, mathematical claims are not true according to these approaches. I believe that versions of the arguments of the paper apply to these, but for reasons of space I do not make that case here.

${ }^{13}$ I should note that I understand the notion of ground in a relatively liberal way: to cover any sort of metaphysical, i.e. non-causal, explanation. For example, I take this notion to cover the explanatory relations that hold between mathematical facts. If one objects to this understanding of the notion, then one can replace my talk of grounding with talk simply of metaphysically explainingnothing essential would be lost. Note in particular that since it is widely accepted that grounding is at least a species of metaphysical explanation, if a given instance does not metaphysically explain a universal generalization, for example, then this instance does not ground the generalization either. Thus, even understood simply in terms of metaphysical explanation, the counterexamples below to the relevant logico-explanatory principles would amount to counterexamples to the widely held principles about grounding.

${ }^{14}$ For such principles see, e.g., Fine [2010] and Rosen [2010].
} 
$(\vee \mathrm{R})$ If $\gamma$, then $\gamma<\beta \vee \gamma$.

( $\forall)$ If $\forall x \varphi$, then $\varphi(x / t)<\forall x \varphi$.

( $\exists$ ) If $\varphi(x / t)$, then $\varphi(x / t)<\exists x \varphi$.

As I noted in the introduction, not only do these seem plausible, they are often used to introduce the notion of grounding. And this plausibility seems just as great in the case of mathematics as elsewhere. For example, it seems that what it is for 17 to be odd and 18 to be even is, on the one hand, for 17 to be odd and, on the other, for 18 to be even. And the natural way of cashing this out would seem to be by saying that the latter two claims ground the former. Similarly, it can seem hard to deny that what it is for every number to be smaller than a prime is (at least in part) for o to be smaller than a prime, for 1 to be, and so on. And again the natural way of articulating this is in terms of ground. But we will see that the strategies of $\$ 1$ lead to violations of these principles-indeed, to cases where they are reversed. This will show that, while we might have hoped that these strategies would be externally revisionary, but internally conservative, they are in fact internally revisionary as well.

Before giving the argument that there are cases where the LE principles are reversed, I should discuss the relationship between a mathematical sentence $\alpha$ and its paraphrase $\alpha_{P}$. On the simplest account of this-and that which I will assumethese sentences express the very same proposition. Thus, in particular, for any $\beta$, $\beta<\alpha$ iff $\beta<\alpha_{P}$, and $\alpha<\beta$ iff $\alpha_{P}<\beta$. On this way of thinking, the logical structure of a mathematical sentence is thus different from that of the proposition it expresses. For example, a conjunction (i.e. a conjunctive sentence) may not express a conjunctive proposition. Nevertheless, one might naturally expect that the LE principles would be preserved (e.g. the propositions expressed by mathematical conjunctions would be grounded by those expressed by their conjuncts). Indeed one might think that this is a precondition on the strategies being well chosen.

However, on an alternative way of thinking about the relationship between a mathematical claim and its paraphrase, the latter rather explains the former, i.e. $\alpha_{P}<\alpha$. On this alternative view, it seems right from the outset that the LE principles fail under the strategies in question. For consider, e.g., $(\wedge \mathrm{L})$. On this way of thinking, $\beta \wedge \gamma$ is grounded (presumably fully) by $(\beta \wedge \gamma)_{P}$. But then it is plausible that this is a complete account of the immediate grounds of $\beta \wedge \gamma$, in which case this will not also be grounded by $\beta$ or by $\gamma$. Of course, there can be no objection in general to a claim having distinct full grounds (cf. disjunctions). But if $\alpha$ is to 
be understood in terms of a paraphrase $\alpha_{P}$, where these are taken to express distinct propositions, then it is surely plausible that every explanation of $\alpha$ should run through $\alpha_{P}$, in which case the latter is the sole immediate ground of the former.

Thus, on the alternative view, the picture would seem to be as follows. There are two levels: paraphrased and paraphrasing. And while there are explanatory connections between the inhabitants of the latter, there are no such between those of the former. Rather, all explanatory connections from paraphrased facts lead to paraphrasing ones. But then $(\wedge \mathrm{L})$ must fail, as must each of the other principles.

Nevertheless, even on this alternative way of thinking, one might expect something close to these principles to hold. Thus, rather than $(\wedge \mathrm{L})$, one would have:

$\left(\wedge \mathrm{L}_{P}\right)$ If $\beta \wedge \gamma$, then $\beta_{P}<(\beta \wedge \gamma)_{P}$.

That is, although the expected connection between conjunctions and conjuncts would not hold at the paraphrased level, its 'shadow' at the paraphrasing level would. And similarly in the cases of the other LE principles.

On this alternative view of the relationship between $\alpha$ and $\alpha_{P}$, versions of the arguments below could still be given, but these would now be aimed not at the original LE principles, but rather at their 'shadows' (in the above sense). These versions of the arguments would show that, far from the paraphrases of logically complex claims always being explained by the paraphrases of logically simpler ones, we sometimes have the exact opposite of this. Thus, although I assume the simple account of the relationship between $\alpha$ and $\alpha_{P}$, the arguments of the paper are, in an important sense, independent of this assumption.

As I said in the introduction, there is a sense in which everything that I argue for is compatible with the orthodox account of logical and explanatory structure, and thus with versions of the LE principles (e.g. versions restricted to claims that do not, in the relevant sense, have paraphrases). The aim is certainly not then to argue that this orthodox account is irredeemable. Insofar as the aim is to argue against anything, it is paraphrase anti-realism. The main aim, however, is simply to establish limits on the extent to which this approach is in the end internally conservative.

\subsection{Fiction Strategy}

In making the case for the reversal of the LE principles, I start with the last strategy of $\$ 1$. The first point to make is that, in general, logically complex claims do not hold in fictions because logically simpler ones do. To illustrate, consider the (very) short story $S$ consisting solely of the following sentence: Holmes heard a knock at 
the door, and Watson answered it. I use $H$ for the first conjunct, $W$ for the second, and $H \wedge W$ for the whole sentence.

Of course, $H, W$ and $H \wedge W$ are all true in $S$, i.e. $\square_{S} H, \square_{S} W$ and $\square_{S}(H \wedge W)$. It seems clear, however, that $H \wedge W$ is not true in $S$ because $H$ and $W$ are. More generally, $H \wedge W$ seems to be a basic truth of $S$ : its being true in $S$ is not explained by other things being true in $S$. Rather, $H \wedge W$ is true in $S$ simply because it is one of the sentences that constitute the story (in this case the only one, of course). In contrast, $H$ does not seem to be a basic truth of $S$. Rather, this is true in $S$ because $H \wedge W$ is. Thus

$$
\begin{aligned}
& \square_{S} H \nless \square_{S}(H \wedge W), \\
& \square_{S}(H \wedge W)<\square_{S} H .
\end{aligned}
$$

Other such examples are easy to generate. For example, suppose that $T$ is a story of the form $\beta, \neg \beta \vee \gamma$. Then $\gamma$ would be true in $T$ because $\beta$ and $\neg \beta \vee \gamma$ are. But $\neg \beta \vee \gamma$ would certainly not hold in $T$ because $\gamma$ does. That is,

$$
\begin{gathered}
\square_{T} \beta, \square_{T}(\neg \beta \vee \gamma)<\square_{T} \gamma, \\
\square_{T} \gamma \nless \square_{T}(\neg \beta \vee \gamma) .
\end{gathered}
$$

Similarly, if a story $R$ consists solely of $\forall x \varphi$, then $\forall x \varphi$ and $\varphi(x / t)$ will both be true in $R$ : but the truth of the former is what explains that of the latter, rather than vice versa.

But when these facts about fictional claims are combined with the strategy of $\$ 1.3$, they yield counterexamples to the LE principles. Recall that, according to that strategy, $\alpha_{P}$ is $\square_{\mathcal{M}} \boldsymbol{\alpha}$. Now consider the following axiom $\boldsymbol{\theta}$ of PA2: ${ }^{15}$

$$
\forall x(N x \rightarrow s x \neq 0)
$$

Of course, $\boldsymbol{\theta}$ is true in the 'number fiction' $\mathcal{M}$. So is its instance $\boldsymbol{\iota}$ :

$$
\text { No } \rightarrow s o \neq 0 .
$$

But-just as in the examples above-it seems clear that $\boldsymbol{\theta}$ is a basic truth of $\mathcal{M}$, whereas $\boldsymbol{\iota}$ is true in $\mathcal{M}$ because $\boldsymbol{\theta}$ is. Thus

$$
\begin{aligned}
& \square_{\mathcal{M}} \boldsymbol{k} \square_{\mathcal{M}} \boldsymbol{\theta}, \\
& \square_{\mathcal{M}} \boldsymbol{\theta}<\square_{\mathcal{M}} \boldsymbol{l} .
\end{aligned}
$$

\footnotetext{
${ }^{15}$ Here $N$ means: is a number; and $s$ is the successor function.
} 
That is, $\iota_{P} \nless \theta_{P}$, but $\theta_{P}<\iota_{P}$. But then, given the assumption that $\theta$ and $\iota$ express the same propositions as their paraphrases, $\iota k \theta$ and $\theta<\iota$, in direct violation of $(\forall)$-indeed, giving exactly the reverse of what $(\forall)$ claims.

For another example, consider again $\iota$, but now in relation to its component $s o \neq 0 .{ }^{16}$ According to $(\vee R), s o \neq 0$ is a partial ground of $\iota$. But, on the strategy under consideration, explanation seems in fact to flow in the opposite direction. For it seems that the reason that $s \mathbf{0} \neq \mathbf{o}$ is true in $\mathcal{M}$ is that $\boldsymbol{\theta}$ (and thus $\boldsymbol{\imath}$ ) is true in $\mathcal{M}$ (together with the fact that $\mathbf{N o}$, an axiom of PA2, is true in $\mathcal{M}$ ). But then $\iota<s o \neq 0$, rather than vice versa. Again, giving not merely the failure, but the reversal, of the relevant LE principle. More examples along these lines are easy to produce (see \$3.1).

\subsection{Consequence Strategy}

As with the fiction strategy, the first point to make here is that, when it comes to logical consequence, claims about the logically complex do not in general hold because claims about the logically simpler do. For example, suppose $\beta$ is $\gamma \wedge \delta$, where $\gamma$ is $G a \wedge G b$ and $\delta$ is $G c \wedge G d$, for a unary predicate $G$ and individual constants $a, b, c$ and $d$.

Of course, $\gamma$ and its conjuncts $G a$ and $G b$ are logical consequences of $\beta$. But explanation flows from the logically complex downwards, rather than vice versa. For the reason $\beta$ entails $G a$, for example, is that it entails $\gamma$, which in turn entails $G a$. In contrast, $\beta$ certainly does not entail $\gamma$ because it entails $G a$. Thus,

$$
\begin{aligned}
& \beta \Rightarrow \gamma<\beta \Rightarrow G a, \\
& \beta \Rightarrow G a \nless \beta \Rightarrow \gamma .
\end{aligned}
$$

Many other examples are available. For instance, $G a$ together with $G a \rightarrow \forall x G x$ of course entails both $\forall x G x$ and $G b$. But it seems that the former fact is the basic one. That is, it seems that the reason

$$
(G a, G a \rightarrow \forall x G x) \Rightarrow G b
$$

holds is that

$$
(G a, G a \rightarrow \forall x G x) \Rightarrow \forall x G x
$$

does, rather than vice versa.

\footnotetext{
${ }^{16} \mathrm{I}$ assume for simplicity that $\beta \rightarrow \gamma$ is defined as $\neg \beta \vee \gamma$. The same point could be made without this assumption, but we would then get a violation not of $(\vee R)$ but of a similar principle about $\rightarrow$.
} 
We then get violations - indeed, reversals — of the LE principles by means of the same examples as in the fiction case. For, given that $\boldsymbol{\theta}$ is one of the sentences that constitutes $\mathcal{M}$, the fact that $\mathcal{M}$ entails $\boldsymbol{\theta}$ is not explained by its entailing anything else. In particular,

$$
\mathcal{M} \Rightarrow \iota \nless \mathcal{M} \Rightarrow \boldsymbol{\theta} .
$$

In contrast, the reason that $\mathcal{M}$ entails $\iota$ does seem to be that it entails $\boldsymbol{\theta}$, i.e.

$$
\mathcal{M} \Rightarrow \boldsymbol{\theta}<\mathcal{M} \Rightarrow \boldsymbol{\iota}
$$

But since, according to this strategy, $\alpha_{P}$ is $\mathcal{M} \Rightarrow \boldsymbol{\alpha}$, this gives $\theta<\iota$ and $\iota \nless \theta$, just as before. Similarly, the reason that $\mathcal{M}$ entails $\boldsymbol{s o} \neq \mathbf{0}$ (the consequent of $\boldsymbol{\iota}$ ), is that it entails $N o$ and $\iota$, i.e. $\iota<s o \neq 0$, rather than vice versa-this time reversing $(\vee R)$.

\subsection{Counterfactual Strategy}

The situation with this strategy is similar to that with the consequence one. Thus, consider again the example of $\beta=\gamma \wedge \delta$, where $\gamma$ is $G a \wedge G b$ and $\delta$ is $G c \wedge G d$. Of course, $\beta \square \mapsto \gamma$ and $\beta \square \rightarrow G a$. But it seems that the former explains the latter rather than the other way around. For what $\beta \square \rightarrow G a$ says is: if it were the case that $\beta$, then $a$ would be $G$. And the reason this holds would seem to be: (i) if it were the case that $\beta$, then it would be the case that $\gamma$; and (ii) $\gamma$ entails $G a$. But here we are explaining $\beta \square \leftrightarrow G a$ using $\beta \square \leftrightarrow \gamma$. This line of thought can then easily be extended-just as with the consequence strategy-to give reversals of the LE principles.

\section{Discussion}

In this section I take up a number of issues raised by the preceding arguments.

\subsection{Other LE Principles}

The first is this. I have given counterexamples to $(\forall)$ and $(v R)$. But what about the other LE principles - how far can the idea of $\$ 2$ be taken? In one sense the answer to this question is straightforward. If one adopts any of the strategies of $\$_{1}$ for numerical claims, then it is hard to see what objection there could be to introducing new mathematical concepts by similar means, i.e. using some theory $\Phi$ of the concepts, in just the way that we used PA2 there. But then, by choosing a theory with 
axioms of the right form, it will be easy to give reversals of the remaining LE principles. For example, if $\Phi$ contains an axiom of the form $\beta \wedge \gamma$, then we will have $(\beta \wedge \gamma)_{P}<\beta_{P}$, i.e. $\beta \wedge \gamma<\beta$, rather than $\beta<\beta \wedge \gamma$-reasoning essentially just as we did in \$2. This is of course a reversal of $(\wedge \mathrm{L})$ (and $\gamma$ would give one of $(\wedge \mathrm{R})$ ). For the existential case, suppose that our theory $\Gamma$ contains the following axioms:

$$
\begin{aligned}
& \delta: \exists x H x \\
& \xi: \forall x(x=e \vee x=j) \\
& \eta: \neg H e .
\end{aligned}
$$

On any of the strategies of $\S_{1}$, we would have $\delta_{P}, \xi_{P}$ and $\eta_{P}$ explaining $(H j)_{P}$, amounting to a reversal of $(\exists)$.

The harder question is whether we can get such additional reversals without resorting to new theories in this way. That is, can we find such counterexamples involving only claims about familiar mathematical objects, such as natural or real numbers or sets (where claims about the latter would be paraphrased using canonical theories such as real analysis or ZFC)? The way that we have produced our counterexamples so far is by focusing on sentences that are either axioms or closely related to them. But we cannot take quite this approach in the case of $(\exists)$ and PA2, for example: simply because $\exists$ does not occur in the axioms of PA2. We would thus have to look at how explanation works, under the strategies of $\$ 1$, when we venture further from their axiomatic foundations. We may indeed be able to find counterexamples to all of the LE principles there-but the investigation of such uncharted waters will not be attempted here.

\subsection{Alternative Theories}

Since we have exploited the specific logical forms of the axioms in giving our counterexamples, it is natural to ask: might we be able to avoid these by using different mathematical theories in our paraphrases, e.g. a different arithmetic theory in place of PA2? In particular, a tempting thought is that we might be able to avoid the reversals by replacing PA2 with a theory consisting only of 'literal' (i.e. atomic or negated atomic) numerical claims.

An immediate problem with this idea is that if $\Sigma$ is the set of true literals of the language of arithmetic, then it will not logically entail claims such as $\boldsymbol{\theta}$ (for $\boldsymbol{\Sigma}$ is

obviously compatible with there being numbers beyond o, 1 etc. and with one of these having o as its successor). But this means that if we replace PA2 with $\Sigma$ in 
our consequence strategy, for example, then $\boldsymbol{\theta}$ would come out as false-clearly an undesirable result. We could try to get around this by strengthening the relevant notion of consequence. One option would be to add the (infinitary) $\omega$-rule: from $\varphi(n)$ for each $n$, infer $\forall x(N x \rightarrow \varphi)$. Or we could add the infinitary axiom

$$
\forall x(N x \rightarrow x=0 \vee x=1 \vee \ldots) .
$$

Either of these fixes would yield the right truth conditions.

The real problem, however, is that this general idea will not extend to claims about other mathematical objects. This is starkest in the case of sets. For the move was viable in the arithmetic case only because we have a name for each number. But we do not of course have a name for each set, nor does there seem to be any acceptable way of extending our language to include one. We might try to insist that every set is a name of itself-so every set would now be a name of our language. But it is hard to see how an anti-realist can help themselves to the resource of such a language. Thus there does not in the end seem to be any way of avoiding the use of logically complex axioms in our paraphrases.

\subsection{Narrow-Scope Explanation}

A very different way of trying to save a version of the LE principles would be to move from wide-scope explanatory claims to narrow-scope ones. Thus, the LE principles we have been discussing are concerned with claims of the form $\beta<\gamma$. In these $<$ takes wider scope than any of operators that occur in $\beta$ or $\gamma$. In particular, if $\beta$ and $\gamma$ are paraphrases offered in $\$_{1}$, then $<$ takes wider-scope than the relevant 'paraphrase operator', i.e. $\Rightarrow, \square_{\mathcal{M}}$ or $\square \rightarrow$. But one way of trying to rescue a version of the principles is to consider instead claims in which $<$ occurs within the scope of the relevant operator. For example, rather than a claim such as

$$
\square_{\mathcal{M}} \iota<\square_{\mathcal{M}} \boldsymbol{\theta},
$$

we would shift attention to

$$
\square_{\mathcal{M}}(\boldsymbol{\iota}<\boldsymbol{\theta}) .
$$

That is, we would paraphrase explanatory mathematical claims in the same way that we paraphrase standard ones. The most straightforward way of implementing this idea would simply be to add the LE principles (i.e. in essentially just the form in which they appear in $\$ 2$ ) to the theory $\mathcal{M}$ that we used in our paraphrases. The LE principles-understood in this narrow-scope way-of course come out as true. 
This tactic seems to do little to reduce the significance of the arguments of $\$ 2$, however. For in thinking about mathematical explanation, it is surely the widescope claims that we are principally interested in. To illustrate, consider the counterfactual strategy. When we pursue the narrow-scope idea, we vindicate the LE principles by turning them into claims about which fundamental mathematical facts would explain which others, if numbers fundamentally existed. The reason that we are not terribly interested in such claims is not principally that they are about which explanatory relationships would hold in a counterfactual situation. After all, on this strategy, mathematics consists of counterfactual claims. The problem with this construal of the LE principles is rather that they concern explanatory relationships between statements made by mathematical language used fundamentally. But when we engage in mathematics (according to the anti-realist views under consideration) we use mathematical language superficially, i.e. to make quite different statements. It is surely the explanatory relationships between these statements (i.e. those we make in the course of standard mathematical practice) that we are principally interested in-but that requires understanding the LE principles in the wide-scope way. And a similar point of course applies to the narrow-scope idea pursued in connection with the other strategies of $\$ 1$.

\subsection{Concrete Objects}

I close by considering the possibility of extending the arguments of the paper to a different sort of object: namely, composite, concrete ones. For many are attracted to an approach to these that, at least in its broad strokes, is similar to the approach to mathematical objects considered above. Thus claims such as 'there are finitely many mangoes', or 'the Yangtze has more bridges than tunnels', would be true, despite the fact that the objects in question - mangoes, rivers, bridges etc.- - are not to be found among the fundamental furniture of the universe. Can our arguments be extended to this sort of approach to concrete objects?

An initial reason for doubting this possibility is that a very different type of paraphrase is often proposed for concrete objects. The idea behind this is that when we say 'there are mangoes', for example, what we are really saying is: there are simples arranged mango-wise. ${ }^{17}$ If this alternative strategy is pursued, then there does not seem to be any prospect of extending the arguments of the paper to this domain.

\footnotetext{
${ }^{17}$ See van Inwagen [1990: 108-14] and Dorr and Rosen [2002].
} 
On the other hand, however, there are some well known drawbacks with this strategy, which might lead to the adoption rather of one along the lines of $\$ 1$ : in which case there would seem to be every reason to expect that the arguments would extend to this case. First, the alternative strategy in terms of simples of course only gets off the ground if mangoes, bridges and so on are in fact composed of these. Indeed, if the strategy is to handle modal discourse, it would seem to require even the impossibility of, for example, bridges that are not composed of simples. But is it not extremely plausible that it is at least possible for there to be objects-such as bridges - all of whose parts themselves have proper parts? Second, this strategy might seem to have a problem with plural quantification - the very device that it exploits. For, under the strategy, apparently singular quantification over composite objects is in fact plural quantification over simples. But what then about plural quantification over composite objects, as in for example the Geach-Kaplan sentence 'some critics admire only one another' (which cannot be understood using only singular quantification)? This would have to be construed as 'superplural' quantification-i.e. over 'pluralities of pluralities'-a device whose coherence has been questioned. ${ }^{18}$ Thus, while the issue is ultimately beyond the scope of this paper, there is at least some reason to think that our arguments might extend to antirealist approaches to composite objects. The upshot would be that in this domain too, the commonly assumed explanatory principles are sometimes the opposite of the truth.

We have seen, then, that a whole range of anti-realist approaches lead to violations, indeed reversals, of standard principles relating logical and explanatory structure. In the end, therefore, these approaches relinquish more than our external judgements. ${ }^{19}$

\section{Appendix: Axioms of PA2}

Here $N$ means: is a number; and $s$ denotes the successor function.

(i) No

(ii) $\forall x(N x \rightarrow N s x)$

\footnotetext{
${ }^{18}$ For this problem with the strategy, see Uzquiano [2004]. For doubts about superplurals in general see, e.g., Lewis [1991: 70-71] or Rumfitt [2005: 102]. (Thanks to [omitted] here.)

${ }^{19}$ [Acknowledgements.]
} 
(iii) $\forall x(N x \rightarrow s x \neq 0)$

(iv) $\forall x \forall y(N x \wedge N y \wedge s x=s y \rightarrow x=y)$

(v) $\forall x(N x \rightarrow x+\mathrm{o}=x)$

(vi) $\forall x \forall y[N x \wedge N y \rightarrow x+s y=s(x+y)]$

(vii) $\forall x(N x \rightarrow x \times \mathrm{O}=\mathrm{o})$

(viii) $\forall x \forall y[N x \wedge N y \rightarrow x \times s y=(x \times y)+x]$

(ix) $\forall X[X \circ \wedge \forall y(N y \wedge X y \rightarrow X s y) \rightarrow \forall y(N y \rightarrow X y)]$

\section{References}

Chihara, C. S. 1973. Ontology and the Vicious-Circle Principle. Ithaca: Cornell University Press.

Dedekind, R. 1888. The Nature and Meaning of Numbers. In Essays on the Theory of Numbers, trans. by W. W. Beman, 21-58. Chicago: Open Court/Project Gutenberg.

Dorr, C. 2008. There Are No Abstract Objects. In T. Sider, J. Hawthorne and D. W. Zimmerman (eds.), Contemporary Debates in Metaphysics, 32-63. Oxford: Blackwell.

Dorr, C. and G. Rosen. 2002. Composition as a Fiction. In R. Gale (ed.), The Blackwell Companion to Metaphysics, 151-74. Oxford: Blackwell.

Field, H. 1989. Realism, Mathematics and Modality. Oxford: Blackwell.

Fine, K. 2010. Some Puzzles of Ground. Notre Dame Journal of Formal Logic 51: 97-118.

Hellman, G. 1989. Mathematics without Numbers: Towards a Modal-Structural Interpretation. Oxford: Clarendon Press.

-. 1996. Structuralism without Structures. Philosophia Mathematica 4: 100-123.

Lewis, D. 1991. Parts of Classes. Oxford: Basil Blackwell.

Putnam, H. 1967. Mathematics without Foundations. Journal of Philosophy 64: 5-22.

Rosen, G. 1990. Modal Fictionalism. Mind 99: 327-54. 
- 2010. Metaphysical Dependence: Grounding and Reduction. In B. Hale and A. Hoffmann (eds.), Modality: Metaphysics, Logic, and Epistemology, 109-35. Oxford University Press.

-. 2011. The Reality of Mathematical Objects. In J. Polkinghorne (ed.), Meaning in Mathematics, 113-31. Oxford University Press.

Rumfitt, I. 2005. Plural Terms: Another Variety of Reference? In J. L. Bermudez (ed.), Thought, Reference, and Experience: Themes from the Philosophy of Gareth Evans, 84123. Oxford: Clarendon Press.

Uzquiano, G. 2004. Plurals and Simples. Monist 87: 429-51.

van Inwagen, P. 1990. Material Beings. Ithaca: Cornell University Press.

Yablo, S. 2001. Go Figure: A Path through Fictionalism. Midwest Studies in Philosophy 25: 72-102. 